\title{
REDTACTON-THE FORWARD THINKING OF HUMAN AREA NETWORK
}

\author{
B.Veera Jyothi ${ }^{1}$, N.Shania Rasheed ${ }^{2}$, V.Mounika ${ }^{3}$ \\ ${ }^{1}$ Assistant Professor, CBIT, India, ${ }^{2,3}$ CBIT, Hyderabad \\ jyothibadnal@gmail.com, shaniarasheed@yahoo.in,mounikaveeramneni@gmail.com
}

\begin{abstract}
Human society is entering an era of ubiquitous computing, when networks are seamlessly interconnected and information is always accessible at our fingertips. In the world of computers, networking is the practice of linking two or more computing devices together for the purpose of sharing data.

Red Tacton is emerging technology that enables the first practical HAN (Human Area Network)between body-centered electronic devices and PCs or other network devices embedded in the environment via a new generation of user interface based on totally natural human actions such as touching, holding, sitting, walking, or stepping on a particular spot. By making Human Area Networks feasible, Red Tacton will enable ubiquitous services based on human centered interactions and therefore more intimate and easier for people to use.

Red Tacton is a break-through technology that uses the surface of the human body as a safe, high speed network transmission path. So we, in this paper are explaining the unique new functional features and enormous potential of Red Tacton as a Human Area networking technology. Technology is making many things easier; we can say that our concept is standing example for that. So far we have seen LAN, MAN, WAN, INTERNET \& many more but here is new concept of "RED TACTON" which makes the human body as a communication network.
\end{abstract}

Keywords: Electro sensor, Human area network, Redtacton

\section{INTRODUCTION}

RedTacton is a new Human Area Networking technology that uses the surface of the human body as a safe, high speed network transmission path. RedTacton uses the minute electric field emitted On the surface of the human body. Technically, it is completely distinct from wireless and infrared.A transmission path is formed at the moment a part of the human body comes in contact with a RedTacton transceiver. Physically separating ends the contact and thus ends communication Using Red Tacton, communication starts when terminals carried by the user or embedded in devices are linked in various combinations according to the user's Communication is possible using any body surfaces, such as the hands, fingers, arms, feet, face, legs or torso. Red Tacton works natural, physical movements. We may have imagined the feature as a place crawling with antennas and emitters, due to the huge growth of wireless communication. And it is seems that the current means of transferring data might already have a very serious competitor none other than the human body.

This technology was developed by Japanese Company Nippon Telegraph and Telephone Corporation. The NTT labs has announced that it is currently testing a revolutionary technology called " red tacton",which uses the electric fields generated by the human body as medium for transmitting the data. The chips which will be embedded in various devices contain a transmitter and receiver built to send and accept data in digital format. The chips can take any type of file such as mp3 music file or mail and convert it in to the format that takes the form of digitals pulse that can be passed and read through a human being electric field the chip in receiver devices reads these tiny changes and convert the file back into its original for.

\section{1) What Is RedTacton??}

It Human society is entering an era of omnipresent computing, when networks are seamlessly interconnected and information is always accessible at our fingertips. The practical implementation of omnipresent services requires three levels of Connectivity.

-Wide Area Networks (WAN), typically via the Internet, to remotely connect all types of severs and terminals;

-Local Area Networks (LAN), typically via Ethernet or WiFi connectivity among all the information and communication appliances in offices and homes;

-Human Area Networks (HAN) for connectivity to personal information, media and communication appliances within the 
much smaller sphere of ordinary daily activities-- the last one meter. NTT's RedTacton is a break-through technology that, for the first time, enables reliable high-speed HAN.

Human Area Networking (HAN) is a technology that safely turns the surface of the human body into a data transmission path at speeds up to $10 \mathrm{Mbps}$ between any two points on the body.Using a novel electro-optic sensor, NTT has already developed a small PCMCIA card-sized prototype RedTacton transceiver.RedTacton enables the first practical Human Area Network between body-centered electronic devices and PCs or other network devices embedded in the environment via a new generation of user interface based on totally natural human actions such as touching, holding, sitting, walking, or stepping on a particular spot. RedTacton can be used for intuitive operation of computer-based systems in daily life, temporary one-to-one private networks based on personal handshaking, device personalization, security, and a host of other applications based on new behavior patterns enabled by Red Tacton. NTT is committed to moving RedTacton out of the laboratory and into commercial production as quickly as possible by organizing joint field trials with partners outside the company.

\section{2) Why Named RedTacton?}

Because with this technology, communication starts by touching (Touch), leading to various actions (Act on) and the colour red to convey the meaning of warmth in communication. Combining these phrases led to the name, "RedTacton".

Meaning Of RedTacton:

TACTON:- "touch-act-on" Meaning "action triggered by touching".

RED:-It is an auspicious colour according to Japanese culture.

\section{3) Previous Work on Electric Field Sensing}

The development of the Human Area Network (HAN) grew out of a meeting between Professor Mike Hawley's Personal Information Architecture Group and Professor Neil Gershenfeld's Physics and Media Group, both at the MIT Media Laboratory. Professor Hawley's group needed a means to interconnect body-borne information appliances, and Professor Gershenfeld's group had been applying electric field sensing to position measurement.

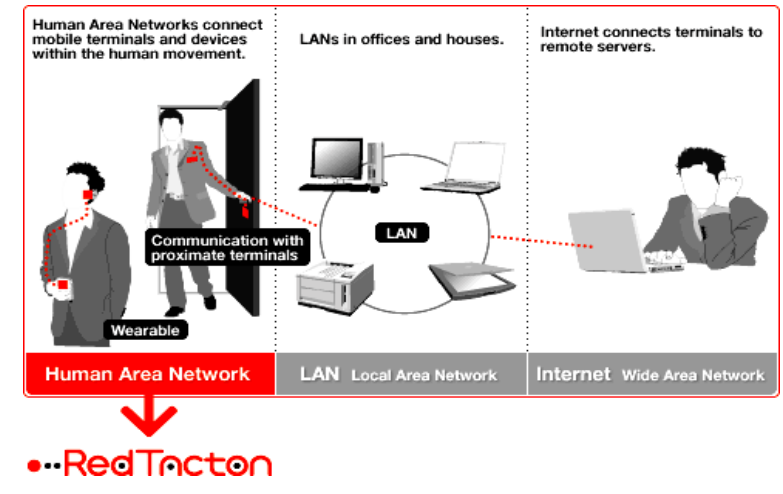

Fig.1.HAN, LAN, WAN

\section{WORKING}

Using a new super-sensitive photonic electric field sensor, Red Tacton can achieve duplex communication over the human body at a maximum speed of $10 \mathrm{mbps}$ The Red Tacton transmitter induces a weak electric field on the surface of the body. The Red Tacton receiver senses changes in the weak electric field on the surface of the body caused by the transmitter .Red tacton relies upon the principle that the optical properties of an electrooptic crystal can vary according to the changes of a weak electric field. Red Tacton detects changes in the optical properties of an electro-optic crystal using a laser and converts the result to an electrical signal in a optical receiver circuit. The transmitter sends data by inducing fluctuations in the minute electric field on the surface of the human body. Data is received using a photonic electric field sensor that combines an electrooptic crystal and a laser light to detect fluctuations in the minute electric field.The naturally occurring electric field induced on the surface of the human body dissipates into the earth. Therefore, this electric field is exceptionally faint and unstable. The photonic electric field sensor developed by NTT enables weak electric fields to be measured by detecting changes in the optical properties of an electro optic crystal with a laser beam.

\section{1) Mechanism of RedTacton}

Data is received using a photonic electric field sensor that combines an electro-optic crystal and a laser light to detect fluctuations in the minute electric field. The naturally occurring electric field induced on the surface of the human body dissipates into the earth. Therefore, this electric field is exceptionally faint and unstable. The photonic electric field sensor developed by NTT enables weak electric fields to be measured by detecting changes in the optical properties of an electro-optic crystal with a laser beam. 


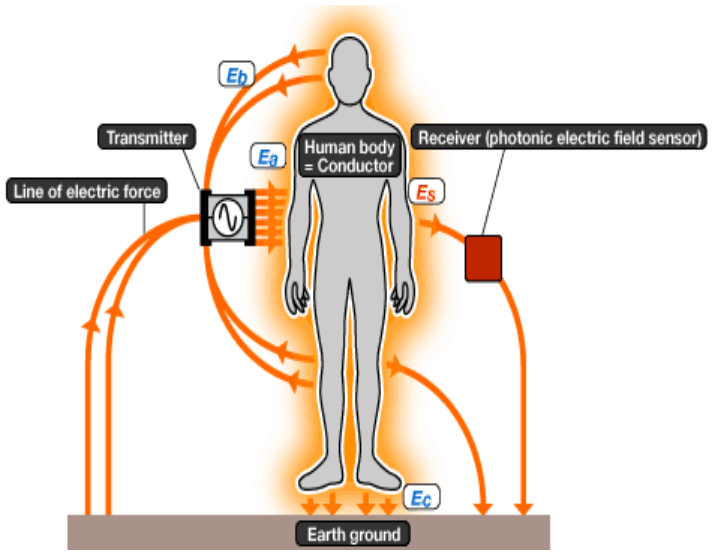

Fig.2.Figure shows the various electric fields on the surface of our body induced by the RED TACTON device.

\section{2) Transmission Steps}

1. The Red Tacton transmitter induces a weak electric field on the surface of the body.

2. The Red Tacton receiver senses changes in the weak electric field on the surface of the body caused by the transmitter.

3. It relies on the principle that the optical properties of the electro-optic crystal varies according to the changes in the weak electric field.

4. It detects the changes in the optical properties of an electrooptic crystal using a laser beam and converts the result into an electrical signal in a detector circuit.

\section{3) RedTacton Transceiver}

- The transmitter consists of a transmitter circuit that induces electric fields toward the body and a data sense circuit, which distinguishes transmitting and receiving modes by detecting both transmission and reception data and outputs control signals corresponding to the two modes to enable two-way communication.

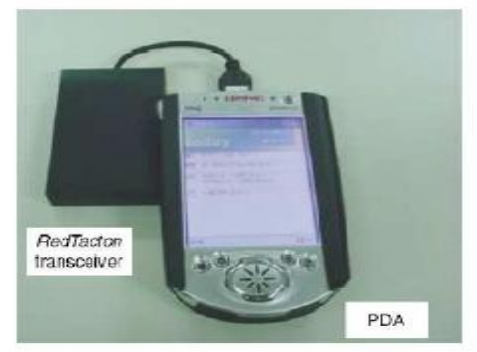

Fig.3. shows a photograph of the RedTacton transceiver connected to a PDA.

-Implementation of receive-first half-duplex communication scheme that sends only after checking to make sure that there is no data to receive in order to avoid packet collisions
-RedTacton takes advantage of the long-overlooked electric field that surrounds the human body.

Figure below shows the block diagram of a RED TACTON transceiver. The signal from the interface is sent to the data sense circuit and the transmitter circuit. The data sense circuit senses the signal and if the data is present it sends control signal to the transmitter which activates the transmitter circuit. The transmitter circuit varies the electric field on the surface of our body. This change in the electric field is detected by the electrooptic sensor. The output of the electro-optic sensor is given to the detector circuit, which in turn given to the interface of the receiving REDTACTON device.

\section{4) Comparison with Other Networks}

The focus on ubiquitous service has brought about the shortening of distances in communication. RedTacton is positioned as the last $1 \mathrm{~m}$ solution to ultimate close-range communication. Wireless communication creates connections when signals arrive, allowing for easy connections because connectors are unnecessary. However, seen from another aspect, the arriving signals can be intercepted, so security becomes an issue.

Wired communication transmits data between two connection points, so interception is difficult and security can be considered to be high. However, connectors and cables are a nuisance. Taking the above points in account, RedTacton is situated directly between wireless and wired communication. In other words, RedTacton allows for easy connection without connectors, while at the same time allowing transmission of data only between two contact points. It thus has the feature of being difficult to intercept.

RedTacton does not require the electrode to be in direct contact with the skin.With the electric amperage method; electrode must be in direct contact with the skin.

\section{PROPERTIES}

\section{System Safety}

NO current flows into human body from RedTacton devices. RedTacton uses the Electric field that occurs naturally on the surface of the human body for Communication. Transmitter and receiver electrodes are covered with an Insulating films. This makes it impossible for current to flow into a person's body from the transceiver. When communication occurs, displacement current is generated by the electrons in the body because the body is subjected to minute electrical fields. However, such displacement currents are very common everyday occurrences to which we are all subjected. RedTacton is in conformity to the "Radiofrequency-exposure Protection" standard (RCR STD-38)" issued by the Association of Radio Industries and Business (ARIB). 


\section{Features Of RedTacton}

RedTacton has three main functional features

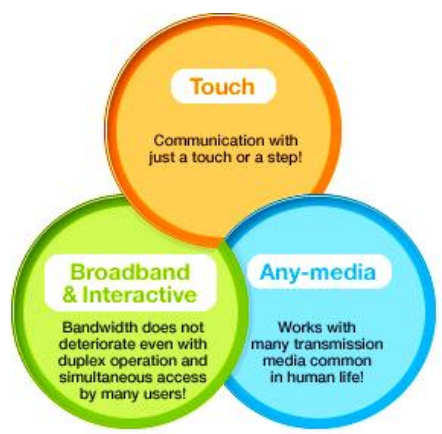

Fig.4.Features of RedTacton

\section{a) Touch:-}

- Communication With Just a Touch Or Step

- Touching, gripping, sitting, walking, stepping and other human movements can be the triggers for unlocking or locking, starting or stopping equipment, or obtaining data. Using RedTacton, communication starts when terminals carried by the user or embedded in devices are linked in various combinations through physical contact according to the human's natural movements.

\section{b) Broadband \& Interactive:-}

Duplex, interactive communication is possible at a maximum speed of 10Mbps. Because the transmission path is on the surface of the body, transmission speed does not deteriorate in congested areas where many people are communicating at the same time. Taking advantage of this speed, device drivers can be downloaded instantly and execute programs can be sent.

\section{c) Any Media:-}

In addition to the human body, various conductors and dielectrics can be used as transmission media. Conductors and dielectrics may also be used in combination.

- A communication environment can be created easily and at low-cost by using items close at hand, such as desks, walls, and metal objects. But there is one limitation on the length of the conductor to be propagated, on installation locations, and on the thickness of the dielectric to be passed through.

\section{APPLICATIONS}

RedTacton has wide variety of applications; some of the applications are as follows:

\section{1) One to One Services:}

-Enable one-to-one services tailored to the user's situation and tastes.
-Attribute information recorded in the RedTacton device is sent to the touched objects.

-The appropriate service is provided based on the attribute information received by the RedTacton receiver

\section{2) Elimination of human error:}

RedTacton devices embedded medicine bottles transmit information on the medicines attributes. If the user touches the wrong medicine, an alarm will trigger on the terminal he is carrying.

The alarm sounds only if the user actually touches the medicine bottle, reducing false alarms common with passive wireless ID tags, which can trigger simply by proximity.

Avoidance of risk at construction sites. (an alarm sounds if special equipment is handled by anyone other than supervisors).

\section{3) Marketing Applications:}

When a consumer stands in front of an advertising panel, advertising and information matching his or her attributes is automatically displayed.

By touching or standing in front of items they are interested in, consumers can get more in-depth information. Inside a shop, shoppers can view related information on their mobile terminals immediately after touching a product.

\section{4) An Alarm:}

Red Tacton devices embedded medicine bottles transmit information on the medicines attributes. If the user touches the wrong medicine, an alarm will trigger on the terminal he is carrying. The alarm sounds only if the user actually touches the medicine bottle, reducing false alarms common with passive wireless ID tags, which can trigger simply by proximity as shown in fig.

\section{5) Intuitive Operations}

Natural movements and actions are the trigger (touch).RedTacton transceivers embedded in two terminals can communicate not only data but also the control or configuration instructions needed to operate devices (broadband \& interactive).

\section{6) Instant Private Data Exchange:}

By shaking hands, personal profile data can be exchanged between mobile terminals on the user.(Electronic exchange of business cards) Communication can be kept private using authentication and encryption technologies. Group photos taken with digital cameras are instantly transferred to individual's mobile terminal. Diagrams written on white boards during meetings are transferred to individual's mobile terminals on the spot. 


\section{7) Personalisation of Mobile Phones:}

Your own phone number is allocated and billing commences. Automatic importing of personal address book and call history. The PC is configured to the user's specifications simply by touching the mouse.

\section{8) Conferencing System:}

An electrically conductive sheet is embedded in the table. A network connection is initiated simply by placing a laptop on the table. Using different sheet patterns enables segmentation of the table into subnets. Walls and partitions can be used as communication media, eliminating construction to install electrical wiring. Ad hoc networking using conductive liquid sprays is possible.

\section{9) Wearable:}

RedTacton can carry music or video between headsets, mobile devices, mobile phones, etc. Users can listen to music from a RedTacton player simply by putting on a headset or holding a viewer.

\section{0) Security Applications}

Automatic user authentication and log-in with just a touch. ID and privileges are recorded in a mobile RedTacton device. Corresponding RedTacton receivers are installed at security check points. The system can provide authentication and record who touched the device, and when.

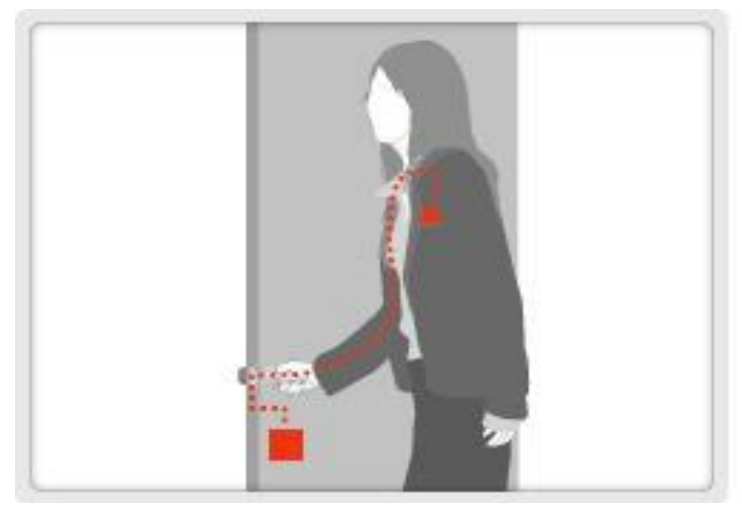

Fig.5. User Verification Management

Carrying a mobile RedTacton-capable device in one's pocket, ID is verified and the door unlocked when the user holds the doorknob normally. Secure lock administration is possible by combining personal verification tools such as fingerprint ID or other biometric in the mobile terminal.

\section{ADVANTAGES AND DISADVANTAGES}

\section{ADVANTAGES:}

i) RedTacton does not require the electrode be in direct contact with the skin. ii.) High-speed communication is possible between two arbitrary points on the body.

iii.) Body-based networking is more secure than broadcast systems ,such as Bluetooth which have high range of about $10 \mathrm{~m}$.

iv.) Network congestion due to fall in transmission speed in multiuser environments is avoided.

v.) Superior than Infrared technology

vi.) Superior than Wi-Fi.

vii.) Data transfer during transfer is less.

viii.) Use of minimum amount of power (of some mill volt range).

ix) Advantage Over Bluetooth:

While it is true that similar personal area networks are already accessible by using radio-based technologies like Wi-Fi or Bluetooth, this new wireless technology claims to be able to send data over the human skin surface at transfer speeds of up to $10 \mathrm{Mbps}$, or better than a broadband T1 connection. Receiving data in such a system is more complicated because the strength of the pulses sent through the electric field is so low.

RedTacton solves this issue by utilizing a technique called electric field photonics: A laser is passed though an electro-optic crystal, which deflects light differently according to the strength of the field across it. These deflections are measured and converted back into electrical signals to retrieve the transmitted data.

\section{DISADVANTAGES:}

i.) It has no compelling applications that aren't already available.

ii.) Too costly.

iii.) It can be useful within centimetres.

iv.) Effects on human body is still under research.

\section{FUTURE DEVELOPMENTS}

RedTacton has a wide range of unique new functional features and enormous potential as a Human Area Networking technology. RedTacton is a big achievement given by NTT to people. NTT is committed to quickly identifying and opening up those application areas with the most commercial promise for RedTacton is a business development process to be coordinated under NTT's Comprehensive Producer Function program.

RedTacton looks remarkably like a big pot of kryptonite is said to allow over $200 \mathrm{kbps}$ of data through the 10 human hands or feet. Telecom giant NTT is planning a commercial launch of a system to enter rooms that frees users from the trouble of rummaging in their pockets or handbags for ID cards or keys.

Data will travel through the user's clothing, handbag or shoes, anyone carrying a special card can unlock the door simply by touching the knob or standing on a particular spot without taking the card out. It will have many future applications such as walkthrough ticket gate, a cabinet that opens only to authorized 
people and a television control that automatically chooses favourite programs. The system also improves security. It ensures that only drivers can open their cars by touching the doors if the keys are in their pockets, not people around them. It uses technology to turn the surface of the human body itself into a means of data transmission.

RedTacton has a wide range of unique new functional features and enormous potential as a Human Area Networking technology. NTT is committed to quickly identifying and opening up those application areas with the the most commercial promise for RedTacton, a business development process to be coordinated under NTT's.

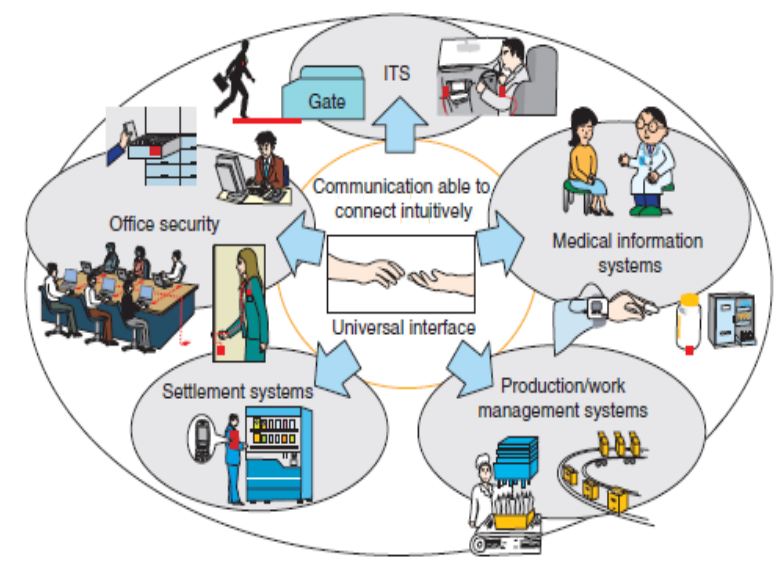

Fig.6.Expected application areas

\section{CONCLUSIONS}

RedTacton is an exciting new technology for human area networking. We have developed a transceiver that uses the human body as a data transmission medium based on an electric-field sensor that uses an electro-optic crystal and laser light. Using this transceiver, we succeeded in achieving 10BASE communication in accordance with IEEE 802.3 through a human body from one hand to the other hand. While our immediate objective is to implement a RedTacton system supporting two way intra body communication at a rate of 10 Mbps between any two points on the body, our longer-term plans include developing a mass-market transceiver interface supporting PDAs and notebook computers while continuing efforts to reduce the size and power consumption of the transceiver to enhance its portability.

NTT is committed to using its comprehensive commercialization functions to push this research out to the marketplace as quickly as possible while moving ahead With tests and trials in collaboration with commercial partners as necessary.
The need for artificial body implants to communicate with each other as well as to report back to a portable device could have quite some value. In fact, according to other researchers, the most important application for body-based networking may well be for these type of communications within, rather than on the surface of, or outside, the body.

RedTacton technology is expected to dominate Bluetooth technology in the future. RedTacton technology could put the use of cables to an end. The problem faced by the RedTacton technology is the cost of development. This technology brings a new dimension of communication which effectively links the user to anyone he wants to communicate. Since it provides high speed communication, it can provide seamless service wherever, whenever and whoever uses it.

This technology definitely stands out with perfection, when transfer of data is fast, feasible and more importantly reliable. So, in few years from now everything is going to fall under this super technology. And, finally I conclude,

\section{"FUTURE BELONGS TO RED TACTON"}

\section{REFERENCES}

[1]. http://www.redtacton.com/en/index.html.

[2]. http://en.wikipedia.org/wiki/RedTacton

[3]. http://www.ntt.co.jp/news/news05e/0502/050218.html

[4]. http://technicalpapers.50webs.com/pdf/redtacton.pdf

[5]. http://www.oppapers.com/essays/Redtacton/166398

[6]. http://www.spectrum.ieee.org/print/2596

[7]. www.masternewmedia.org

[8]. http://www.oppapers.com/essays/Redtacton/166398. 